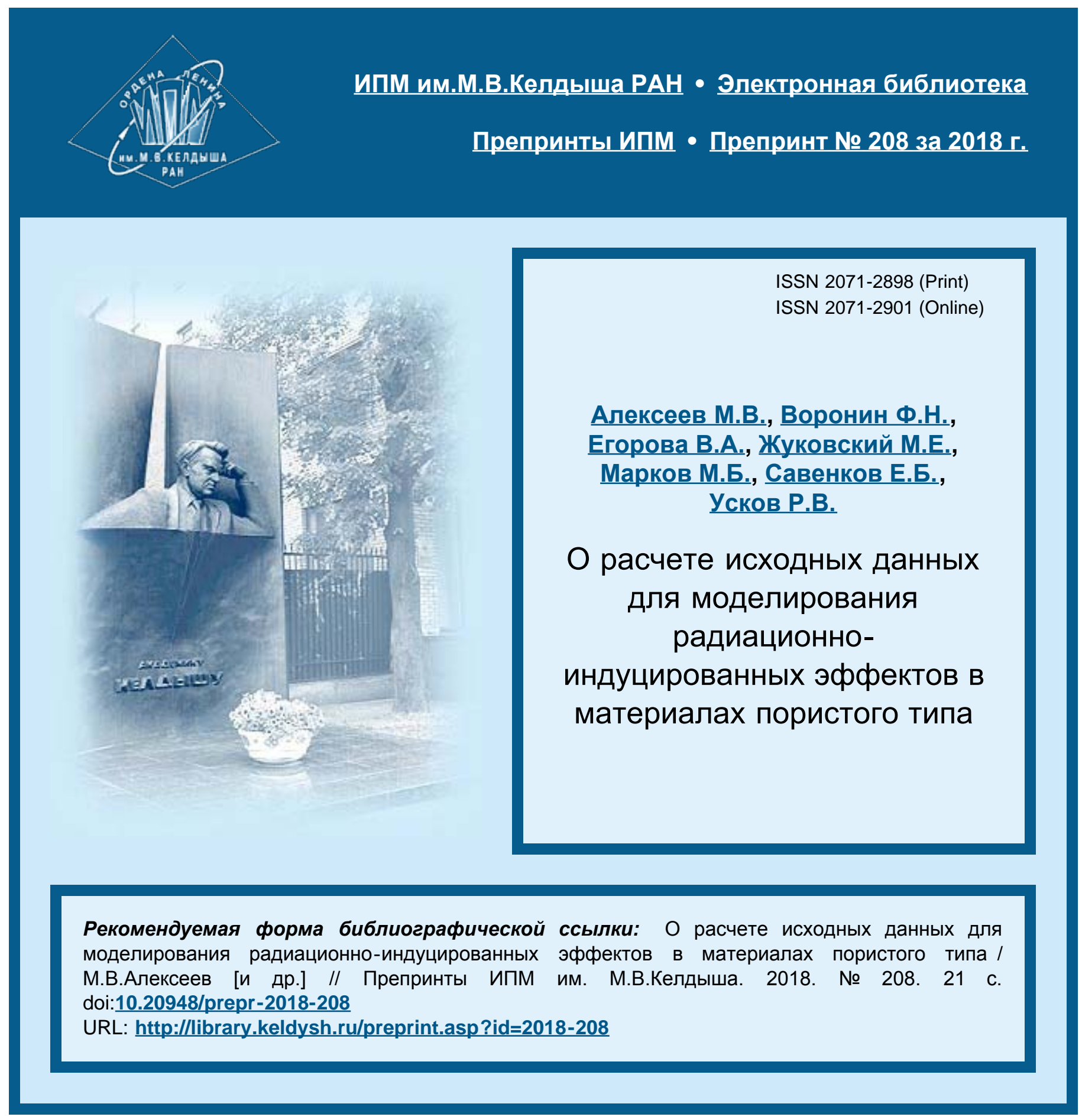




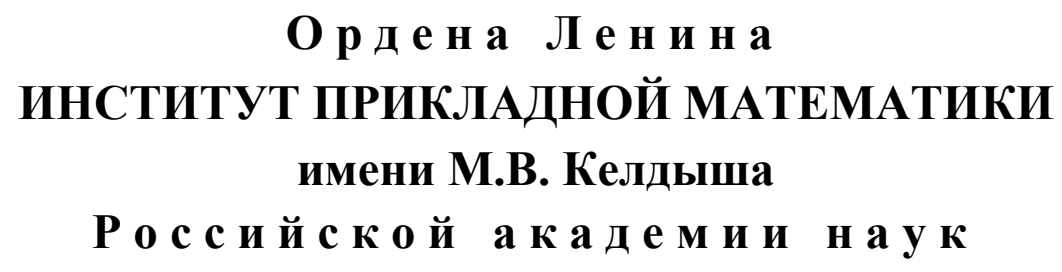

М.В. Алексеев, Ф.Н. Воронин, В.А. Егорова, М.Е. Жуковский, М.Б. Марков, Е.Б. Савенков, Р.В. Усков

\author{
О РАСЧЕТЕ ИСХОДНЫХ ДАННЫХ \\ ДЛЯ МОДЕЛИРОВАНИЯ \\ РАДИАЦИОННО-ИНДУЦИРОВАННЫХ ЭФФЕКТОВ \\ В МАТЕРИАЛАХ ПОРИСТОГО ТИПА
}

Москва - 2018 
М.В. Алексеев, Ф.Н. Воронин, В.А. Егорова, М.Е. Жуковский, М.Б. Марков, Е.Б. Савенков, Р.В. Усков

О расчете исходных данных для моделирования радиационноиндуцированных эффектов в материалах пористого типа

Разработан подход к расчету исходных данных для моделирования радиационно-индуцированных термомеханических эффектов в гетерогенных материалах пористого типа. Подход основан на многомерной аппроксимации результатов моделирования взаимодействия излучения с веществом на разностную сетку, предназначенную для решения уравнений термодинамики. В качестве метода аппроксимации применяется модель нелинейной регрессии. Построена физико-геометрическая модель пористой среды с прямым учетом её микроструктуры. Представлены результаты модельного расчета термомеханических эффектов в пористой преграде, облучаемой потоком рентгеновских фотонов.

Ключевые слова: перенос излучения, пористые среды, многомерная аппроксимация.

Mikhail Vladislavovich Alekseev, Fedor Nikolaevich Voronin, Varvara Alekseevna Egorova, Mikhail Evgenievich Zhukovskiy, Mikhail Borisovich Markov, Evgeniy Borisovich Savenkov, Roman Vladimirovich Uskov

\section{On computing the initial data for modeling of radiation-induced effects in porous materials}

An approach to the calculation of initial data for modeling radiation-induced thermomechanical effects in heterogeneous porous materials is developed. The approach is based on the multidimensional approximation of the results of modeling the interaction of radiation with matter on a difference grid designed to solve the thermodynamics equations. The nonlinear regression model is used as an approximation method. A physical and geometric model of the porous medium with a direct account of its microstructure is constructed. The results of the model calculation of thermomechanical effects in a porous barrier irradiated by $\mathrm{x}$-ray photon flux are presented.

Keywords: radiation transport, porous media, multi-dimensional approximation. Исследование выполнено при поддержке гранта РФФИ № 18-01-00582 


\section{Оглавление}

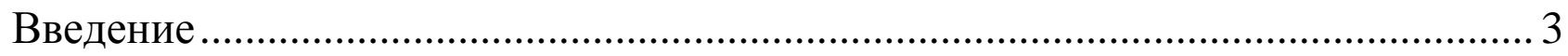

1. Моделирование комплексных процессов взаимодействия излучения с гетерогенными материалами 4

2. Интеграция «по данным» результатов статистического моделирования переноса излучения и методов численного решения задач термодинамики.......6

2.1 Геометрическая модель пористой среды ...................................................... 7

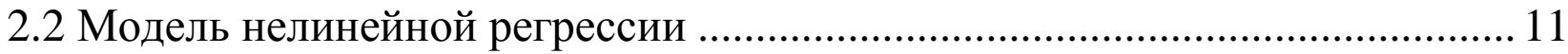

2.3 Пример аппроксимации энерговыделения излучения в пористом

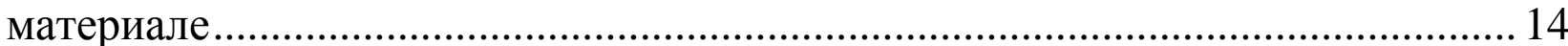

3. Результаты моделирования радиационно-индуцированных

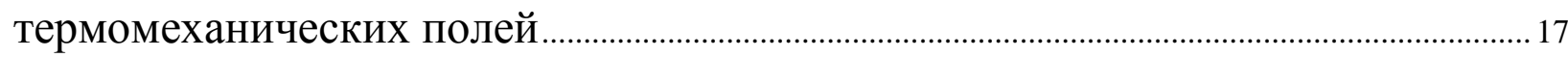

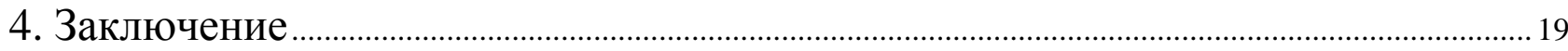

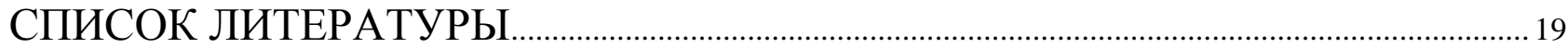

\section{Введение}

Пористые материалы широко применяют в машиностроении, теплоэнергетике, ракетной, авиационной, химической и других отраслях промышленности. Это обусловлено тем, что эти материалы обеспечивают требуемые прочностные, теплофизические, гидравлические, технологические свойства и способны работать при высоких температурах и давлениях. Такие материалы применяют в системах теплозащиты ракетных двигателей [1], их используют для создания оболочек турбинных лопаток парогазовых установок и газотурбинных двигателей $[2,3]$.

В современной инженерной практике материалы защитных покрытий чаще всего являются композиционными с пористой структурой. Именно сложная гетерогенная структура материала обеспечивает сохранение его свойств в условиях многофакторного воздействия ионизирующего излучения, в том числе воздействия радиационно-индуцированных термомеханических эффектов.

Главная проблема состоит в определении состава и микроструктуры материала, обеспечивающих ему заданные макроскопические свойства. Современная практика решения этой задачи состоит в применении упрощенных гомогенизированных математических моделей, инженерных методик расчета характеристик взаимодействия излучения с плоско-слоистыми преградами и проведении большого числа лабораторных экспериментов для определения состава фаз гетерогенного вещества, геометрических параметров микроструктуры материала и т.д. Такой подход является дорогостоящим и требует больших временных ресурсов и материальных затрат. Кроме того, он имеет принципиальные огра- 
ничения, связанные с отсутствием универсальных методик определения макроскопических свойств материала и возможности анализа «редких», но катастрофических событий (электрический пробой, разрушение материала и пр.).

Структура современных гетерогенных веществ настолько сложна, что необходим прямой учет пористости материала в совокупности с целым набором физических факторов воздействия. Единственным перспективным путем решения проблемы в настоящее время является математическое моделирование комплексных механизмов воздействия излучения на основе применения фундаментальных математических моделей с прямым учетом микроструктуры материала.

В настоящее время основной принцип моделирования свойств пористых материалов заключается в том, что сложный реальный объект заменяют более простой геометрической моделью, доступной для элементарного математического описания. При таком упрощении игнорируются многие особенности пористой структуры, модель содержит только главные определяющие черты пористого тела. Такие модели непригодны для анализа процессов, протекающих в масштабе пространственных неоднородностей материала (разрушение или заполнение пор композита, электрический пробой и ряд других).

Адекватное математическое моделирование распространения излучения и генерации вторичных термомеханических эффектов в материале пористого типа возможно только с прямым учетом его структуры на микроуровне.

Настоящая публикация является продолжением работ по построению моделей и алгоритмов компьютерного моделирования воздействия ионизирующего излучения и вторичных радиационно-индуцированных эффектов на гетерогенные материалы сложной внутренней структуры. В работе [4] предложены способы построения физико-геометрической модели распространения излучения в пористом материале с прямым учетом его структуры на микроуровне.

В настоящей работе рассмотрена процедура переноса результатов статистического моделирования энерговыделения излучения на разностную сетку, обеспечивающая интеграцию «по данным» для различных групп уравнений модели генерации вторичных, радиационно-индуцированных эффектов.

\section{1. Моделирование комплексных процессов взаимодействия}

\section{излучения с гетерогенными материалами}

Рассматриваемый в настоящей работе модельный сценарий взаимодействия излучения с материалом включает в себя следующие элементы. Рассматривается существенно неоднородный материал, на который от внешнего источника «падает» достаточно сильный импульс ионизирующего излучения. В процессе распространения излучения в объекте часть его энергии поглощается веществом. Это приводит к нагреву материала и, как следствие, быстрому развитию в нем сильно неоднородных полей температуры и давления. При достаточно высокой интенсивности излучения процесс нагрева протекает очень быстро и близок к «ударному» (то есть время нагрева сравнимо со звуковыми времена- 
ми в материале). Одновременно с этим начинается процесс релаксации возникших в материале термомеханических полей, в результате которого может происходить деградация функциональных характеристик материалов и конструкций, в частности развитие повреждений и разрушение в пространственном масштабе пор материала.

Комплексная вычислительная методика анализа описанного выше модельного сценария должна включать в себя следующие компоненты:

- способы описания и средства построения геометрической модели среды с прямым разрешением ее микроструктуры [4];

- вычислительные алгоритмы моделирования процессов развития фотонэлектронного каскада $[5,6]$, приводящего к энерговыделению излучения в пористой среде, облучаемой ионизирующим излучением;

- математические модели и алгоритмы генерации и развития вторичных радиационно-индуцированных термодинамических и термомеханических процессов [7] в материале;

- эффективные средства интеграции «по данным» между указанными классами моделей.

Основное внимание в настоящей работе уделено выбору и построению способов интеграции «по данным» результатов статистического моделирования переноса излучения в пористых средах и исходных данных для численного решения задач термомеханики. Такие способы предложено строить на основе методов многомерной аппроксимации.

Исследования взаимодействия излучения с веществом и возникновение вторичных эффектов проводят с применением различных физикоматематических моделей и вычислительных алгоритмов, а также разных дискретных геометрических описаний объекта и расчетной области. При моделировании развития фотон-электронного каскада облучаемые объекты описываются с помощью граничных поверхностей (оболочек), ограничивающих гомогенные составляющие объекта $[6,8]$, а в качестве регистрирующей системы используется, как правило, совокупность равномерно распределенных детекторов различной формы, в которых рассчитываются функционалы на пространстве решений уравнения переноса [9]. Термодинамические и термомеханические процессы моделируют, как правило, на прямоугольных пространственных декартовых разностных сетках $[10,11]$. Термодинамические эффекты могут моделироваться на неструктурированных пространственных сетках [10-12].

Вопросы применения различных дискретных моделей объекта и расчетной области рассмотрены, например, в работах $[13,14]$. В этих работах обосновывается необходимость решения задачи аппроксимации данных с одной геометрической структуры (дискретной системы детекторов частиц излучения) на другую (термодинамическую декартову разностную сетку). В задаче моделирования радиационно-индуцированных термомеханических эффектов значения объемной плотности энерговыделения, полученные на множестве равномерно распределенных внутри объекта объемных детекторов в результате статистическо- 
го моделирования каскадных процессов переноса излучения [6], требуется аппроксимировать на разностную сетку для расчета вторичных эффектов термодинамики.

Общая схема методики имеет следующий вид:

«Препроиессинг»

Шаг 1. Построение дискретной модели пористой среды.

Шаг 2. Построение системы детекторов для статистической оценки энерговыделения излучения в веществе.

Шаг 3. Построение декартовой ортогональной («воксельной») сетки для расчета динамики термомеханических полей в материале.

«Расчет»

Шаг 4.

a) Статистическое моделирование взаимодействия излучения с пористой средой и оценка энерговыделения в построенной системе детекторов.

б) Перенос значений энерговыделения на «термомеханическую» расчетную сетку для инициализации термомеханического состояния среды.

Шаг 5. Расчет динамики термомеханических полей, в том числе перераспределения вещества в пространстве.

Современные пористые материалы имеют сложную внутреннюю структуру, что заметно осложняет выбор типа аппроксимации и метода построения аппроксимирующих функций. В работе [14] вопросы многомерной аппроксимации данных применительно к решению обсуждаемых задач предлагается рассматривать в рамках технологии машинного обучения. Введена классификация типов многомерной аппроксимации в зависимости от характера физической величины, которую необходимо аппроксимировать, от геометрии и свойств материала облучаемого объекта, а также от характеристик источника излучения.

\section{2. Интеграция «по данным» результатов статистического моделирования переноса излучения и методов численного решения задач термодинамики}

Математическое моделирование радиационно-индуцированных термодинамических эффектов подразумевает использование результатов моделирования первичного физического процесса (взаимодействия излучения с веществом) для численного исследования вторичных (радиационно-индуцированных) процессов. В связи с этим ставится задача многомерной аппроксимации данных с одной трехмерной геометрической структуры на другую.

Для многомерной аппроксимации применительно к решению ряда вышеупомянутых задач предлагается применить методы машинного обучения, а именно технологию искусственных нейронных сетей и модель нелинейной регрессии [14].

В работе [14] определены три типа аппроксимации применительно к задачам моделирования комплексного воздействия ионизирующего излучения: 
- глобальная аппроксимация, которая подразумевает поиск одной аппроксимирующей функции для физической величины во всем объекте (на всей области определения искомой функции);

- локальная аппроксимация, в основе которой лежит разбиение рассматриваемой области (объекта) на подобласти, в каждой из которых строится своя аппроксимирующая функция;

- локальная аппроксимация по целевому множеству, особенность которой заключается в построении аппроксимирующей функции для каждой точки множества («целевое» множество), на которое требуется «перенести» известные (рассчитанные) данные («обучающее» множество).

Подробно особенности построения многомерной аппроксимации первых двух типов рассмотрены в работе [14]. Основное отличие глобальной и локальной аппроксимации от локальной аппроксимации по целевому множеству заключается в том, что первые два типа опираются на априорную информацию об аппроксимируемой функции и геометрическую структуру исходного объекта, их построение проводится независимо от целевого множества $[13,14]$, в то время как третий тип аппроксимации «привязан» главным образом к структуре и расположению точек целевого множества.

Важным фактором при решении вопроса о выборе способа аппроксимации является наличие и количество экстремумов у функции, описывающей исследуемую физическую величину, а также априорная информация о гладкости этой функции.

В работе [14] подробно рассмотрены способы многомерной аппроксимации для случаев, когда воздействию ионизирующего излучения подвергается однородная среда (глобальная аппроксимация) или гетерогенный материал регулярной замкнуто-ячеистой структуры (локальная аппроксимация). Для таких материалов эффективным оказывается применение технологии нейронных сетей (многослойный персептрон).

В случае, когда аппроксимируемая функция не обладает гладкостью и имеет большое количество экстремумов, расположение которых неизвестно, эффективным является применение локальной аппроксимации по целевому множеству. Такая аппроксимация чувствительна к сильным изменениям на коротких пространственных промежутках и способна их правильно учесть, что особенно важно для стохастических структур, например для гетерогенных сред пористого типа. В основе этого способа аппроксимации лежит модель нелинейной регрессии [15]. Этот подход и способ его реализации применительно к рассматриваемым задачам рассмотрены в настоящей работе.

\section{1 Геометрическая модель пористой среды}

В качестве объекта исследования рассматривается пористый материал с изолированными порами. В общем случае его структура определяется распределением пустот по размерам, форме и ориентации, а также объемной долей пустот - пористостью среды. В настоящей работе рассматривается модельная 
среда с порами сферической формы и одинакового диаметра. В качестве параметров геометрической структуры среды задаются диаметр поры и средняя пористость образца материала.

Для построения геометрической модели материала, то есть конкретного размещения внутри образца материала пустот с заданными геометрическими свойствами, могут быть использованы различные алгоритмы. Наиболее популярным из них является алгоритм Любачевского-Стилинжера [16 - 18]. Входными данными алгоритма является число «частиц» (пор) и их финальный диаметр. В начальный момент времени считается, что частицы равномерно распределены в заданном объеме, имеют нулевой радиус и взаимодействуют посредством заданного короткодействующего потенциала парного взаимодействия (в простейшем случае - потенциала «твердой стенки»). Эволюция положения частиц описывается системой уравнений динамики взаимодействующих точек. Начальное распределение скоростей частиц является заданной случайной величиной. В ходе эволюции системы происходит медленный рост размера частиц по заданному закону, причем размер частиц учитывается при расчете их столкновений. Траектории частиц в общем случае будут являться хаотическими. В результате, при достаточно медленном изменении размеров частиц, алгоритму «удается» разместить их в пространстве без пересечений [4].

Результаты моделирования микроструктуры материала показаны на рис.1. Приведен пример размещения пустот размером 50 мкм в образце $1 \times 1 \times 0.25$ мм. Пористость материала в этом примере составила 40\%. Количество пор около 4000 .

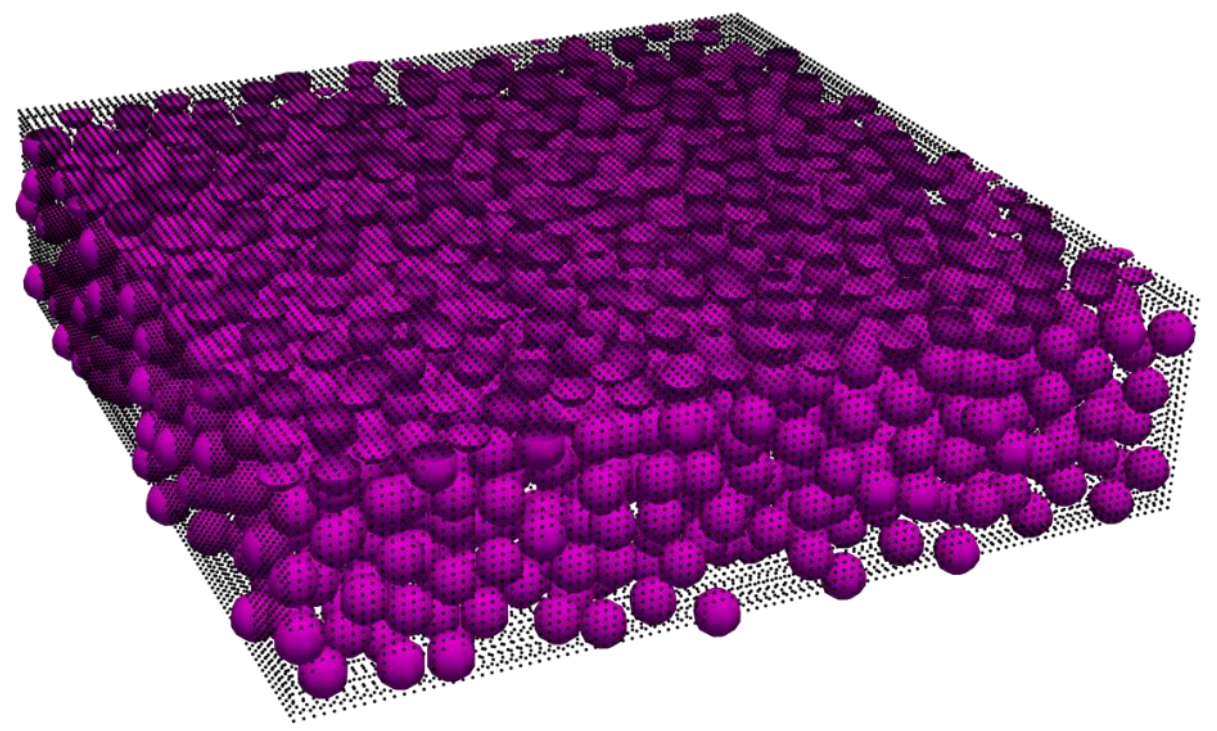

Рис. 1. Расположение пустот в пластине пористого материала

Задача моделирования прохождения излучения через вещество заключается в вычислении показаний «детекторов», в которых определяется измеряемая (искомая) величина. Поэтому регистрирующая система (система детекторов, определяемая типом искомой величины) является неотъемлемой составной частью геометрического описания среды. 
Внутри пластины строится детектирующая система, детекторы которой разных размеров имеют сферическую форму [4]. Расположение детекторов в объекте приведено на рис. 2.

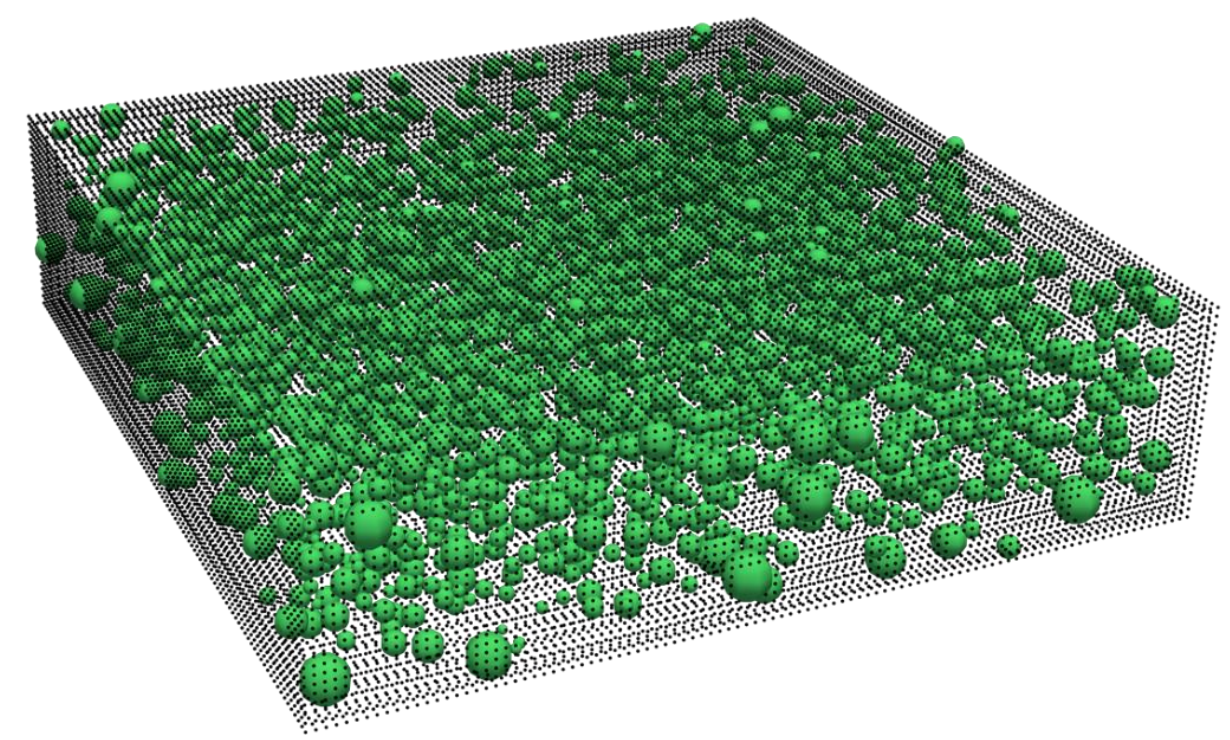

Рис. 2. Расположение детекторов для расчета энерговыделения излучения

Распределение детекторов по размерам показано в виде гистограммы на рис. 3. Детекторы не пересекаются между собой и лежат целиком внутри наполнителя (не пересекаются с порами).

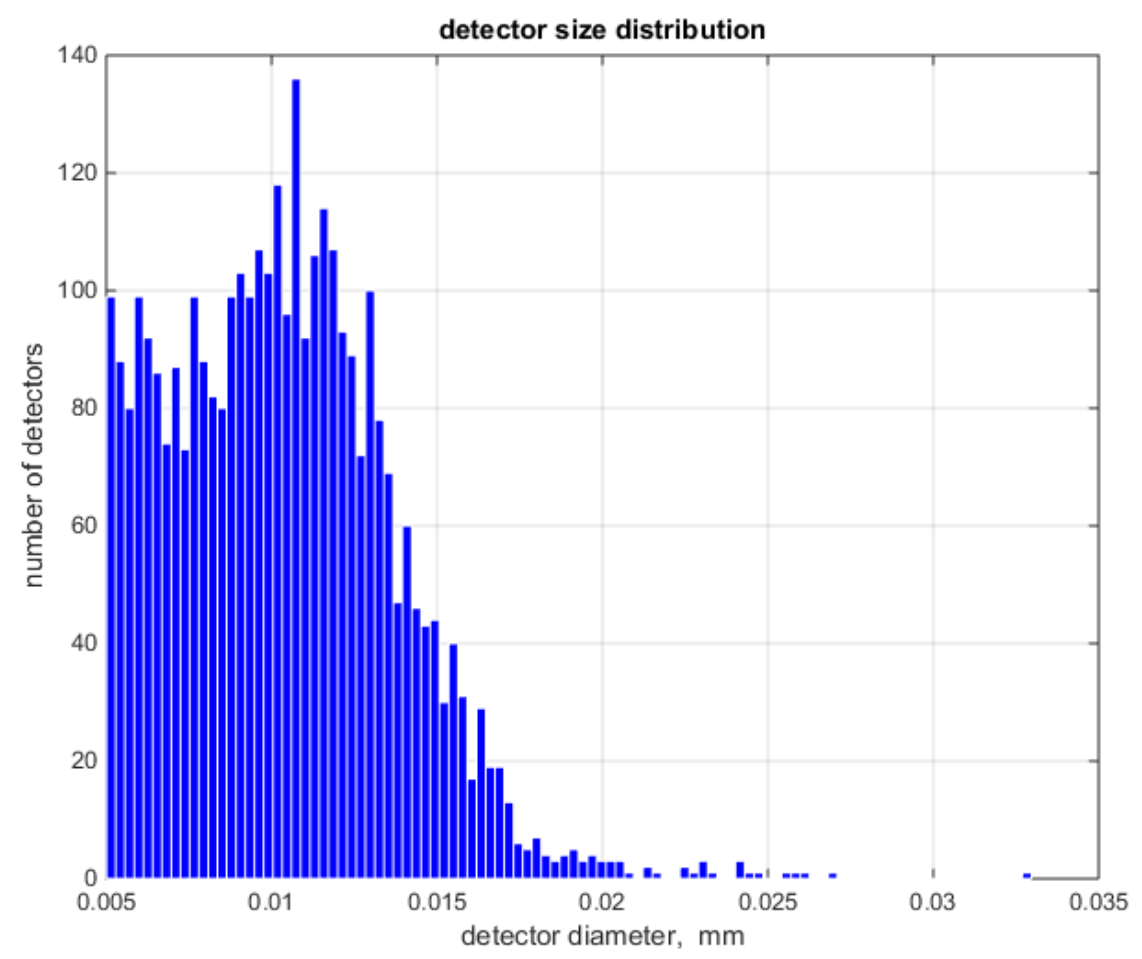

Рис. 3. Распределение детекторов по размеру

На рис. 4 изображено взаимное расположение пор и детекторов. 


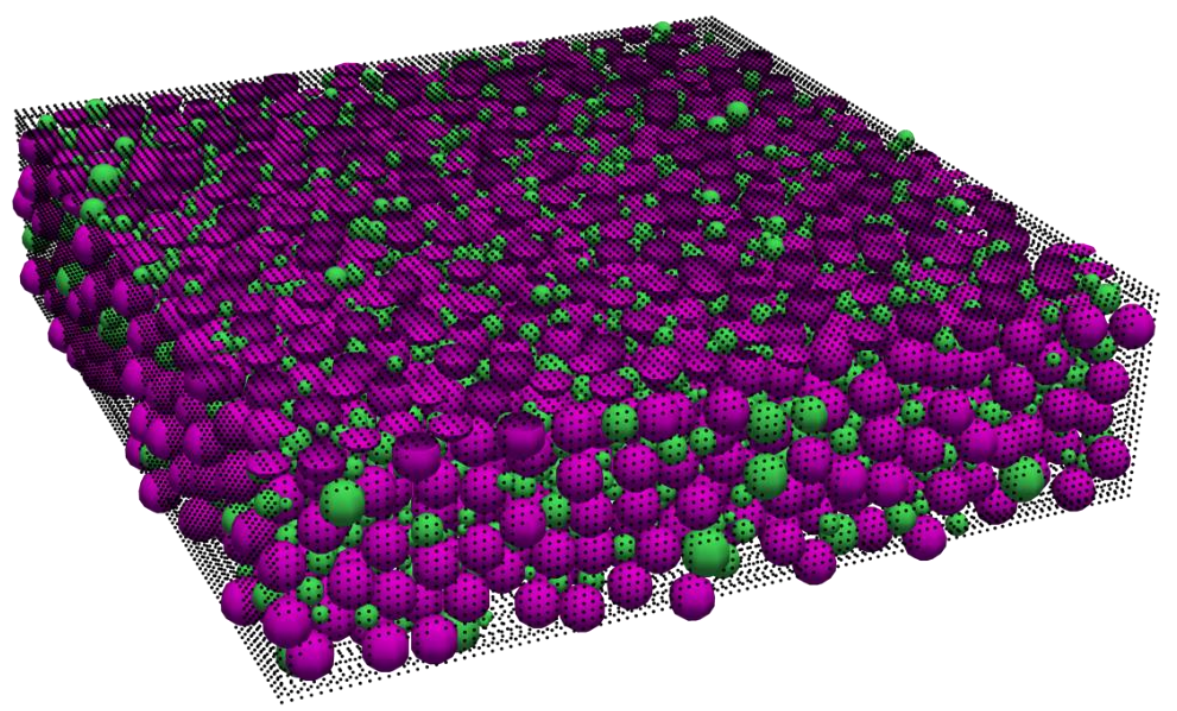

Рис. 4. Взаимное расположение пор и детекторов

С использованием построенной детектирующей системы моделируется процесс переноса излучения методом Монте-Карло [5, 6].

Множество центров детекторов (в данном примере около 3500 точек) вместе с соответствующими рассчитанными значениями плотности энерговыделения являются обучающим множеством. Значения плотности энерговыделения в относительных единицах, рассчитанные в центрах детекторов, показаны на pис.5. Изображение на уровне $\mathrm{z}=0.0095$ см от грани, перпендикулярно которой в объект входит излучение, построено с помощью триангуляции Делоне [19].

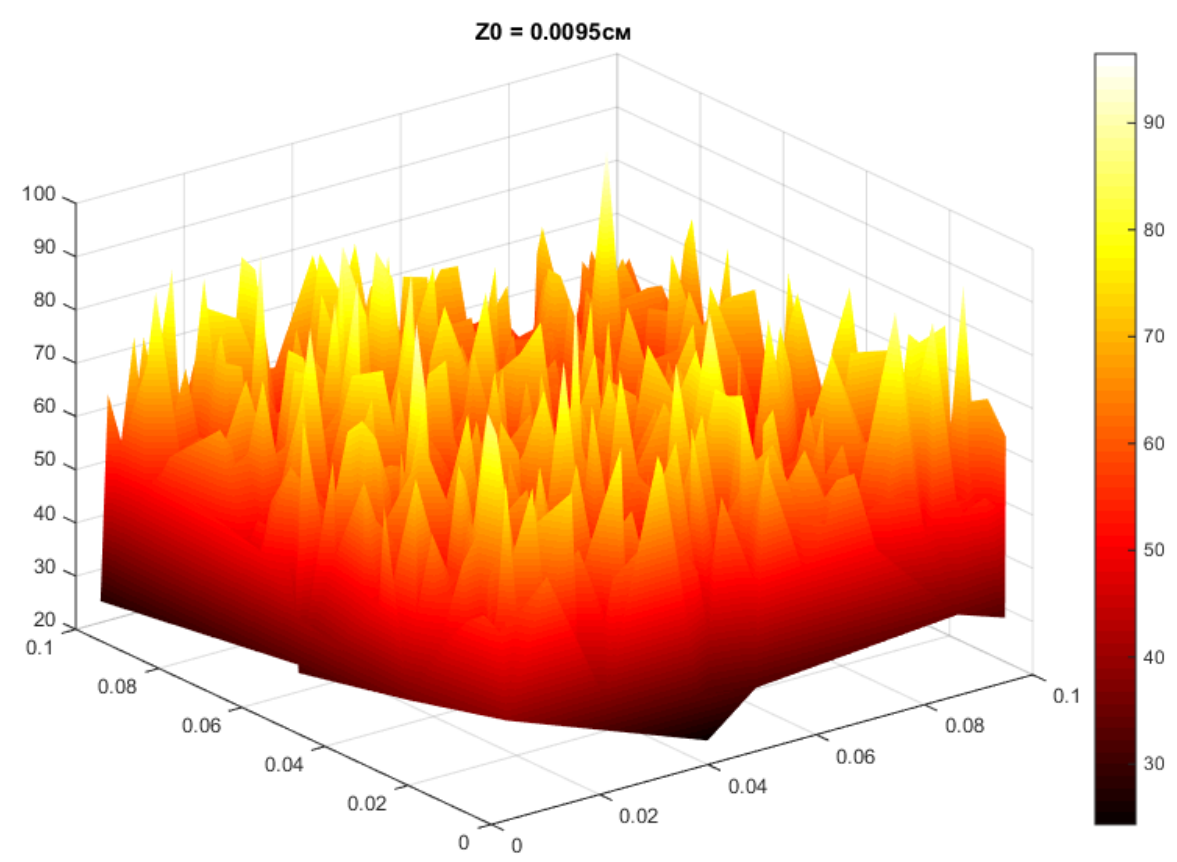

Рис. 5. Значения плотности энерговыделения в центрах детекторов, находящихся на глубине $\mathrm{z}=0.0095 \mathrm{~cm}$

Изображенная на рис. 5 структура обучающего множества (огромное ко- 
личество экстремумов рассчитанной функции энерговыделения, стохастическое множество детекторов) не позволяет применить глобальный или локальный тип аппроксимации [14].

Целевым множеством в рассматриваемом примере выбрана совокупность центров ячеек пространственной регулярной разностной сетки с шагом $0.001 \mathrm{~cm}$ по каждой из переменных $(x, y, z)$, общее количество ячеек -250000.

Для построения аппроксимирующей функции в гетерогенных материалах пористого типа разработан вариант локальной аппроксимации по цеелевому множеству с применением модели нелинейной регрессии.

\section{2 Модель нелинейной регрессии}

Нелинейная регрессия - это разновидность регрессионного анализа, в которой зависимость между случайными величинами моделируется нелинейной функцией с неизвестными параметрами по эмпирическим (наблюдаемым) данным.

В качестве эмпирических данных будем рассматривать результаты статистического расчета физической величины $f_{m}\left(\mathbf{r}_{m}\right), m=1, \ldots M$, (например, энерговыделения излучения) на некотором множестве точек $\mathbf{r}_{m}=\left(x_{m}, y_{m}, z_{m}\right) \in \Omega, m=1, \ldots M$.

Требуется построить аппроксимацию функции $f$ для любого $\mathbf{r} \in \Omega$.

В терминах регрессионного анализа переформулируем задачу аппроксимации следующим образом.

Пусть имеется упорядоченная пара случайных величин $(\eta, \xi)$. Необходимо найти зависимость между $\eta$ и $\xi$. В качестве искомой зависимости выбираем условное математическое ожидание

$$
M(\eta \mid \xi=\mathbf{r})=M(\eta \mid \mathbf{r})=f(\mathbf{r}) .
$$

Если $g_{\eta}(\eta \mid \mathbf{r})$ - условная плотность распределения вероятности величины $\eta$, то

$$
M(\eta \mid \mathbf{r})=f(\mathbf{r})=\int_{-\infty}^{\infty} y g_{\eta}(y \mid \mathbf{r}) d y .
$$

Функция $f(\mathbf{r})$ называется регрессией величины $\eta$ на $\xi$.

Среднеквадратичной погрешностью оценки величины $\eta$ по наблюдаемому значению $\mathbf{r}$ случайной величины $\xi$ является условная дисперсия

$$
D(\eta \mid \mathbf{r})=\int_{-\infty}^{\infty}(y-f(\mathbf{r}))^{2} g_{\eta}(y \mid \mathbf{r}) d y .
$$

Для определения погрешности регрессионной оценки во всем диапазоне изменения $\xi$ величину $D(\eta \mid \mathbf{r})$ нужно усреднить. Если плотность распределения величины $\xi$ есть $g_{\xi}(\mathbf{r})$, то 


$$
\delta=\int_{-\infty}^{\infty} D(\eta \mid \mathbf{r}) g_{\xi}(\mathbf{r}) d \mathbf{r}=\iint_{-\infty}^{\infty}(y-f(\mathbf{r}))^{2} g_{\eta}(y \mid \mathbf{r}) g_{\xi}(\mathbf{r}) d \mathbf{r} d y=M(\eta-f(\mathbf{r}))^{2} .
$$

Рассеяние $\delta$ минимально при $\eta=f(\mathbf{r})$ (в центре рассеяния). Таким образом, регрессия $\eta$ на $\xi$ является наилучшей (в смысле минимума среднеквадратичного отклонения) оценкой зависимости $\eta$ от $\xi$.

В случае, когда регрессия неизвестна, оценку зависимости $\eta$ от $\xi$ ищут в некотором классе функций $F(\xi, \theta)$ случайной величины $\xi$. Величина $\theta$ - это вектор неизвестных параметров функции $F$ (например, если рассматривается класс квадратичных функций $F(\xi)=\alpha \xi^{2}+\beta \xi+\gamma$, то вектор $\left.\theta=(\alpha, \beta, \gamma)\right)$.

Для нахождения наилучшей оценки зависимости $\eta$ от $\xi$ функцию $F$ необходимо минимизировать по вектору параметров $\theta$. Условное математическое ожидание

$$
M(\eta-F(\xi, \theta))^{2}=Q(\theta) .
$$

После нахождения компонент вектора параметров $\theta_{\min }$, доставляющих минимум величине $Q$, зависимость между величинами $\xi$ и $\eta$ определяется соотношением $\eta=F\left(\xi, \theta_{\min }\right)$.

На практике известными являются только совокупности наблюдаемых значений $\left\{\mathbf{r}_{m}\right\}_{m=1}^{M}$ и $\left\{y_{m}\right\}_{m=1}^{M}$ случайных величин $\xi$ и $\eta$. Таким образом, требуется найти значения параметров $\theta$, которые доставляют минимум величине

$$
Q_{M}(\theta)=\sum_{m=1}^{M}\left(y_{m}-F\left(\mathbf{r}_{m}, \theta\right)\right)^{2}=\sum_{m=1}^{M} q_{m}{ }^{2} .
$$

Величину $Q_{M}$ называют суммой регрессионных остатков.

Пусть вектор параметров $\theta$ содержит $N$ компонент: $\left\{\theta_{n}\right\}_{n=1}^{N}$. Для нахождения минимума величины $Q_{M}$ применяют следующую процедуру.

Приравнивают к нулю первые частные производные функции $Q_{M}$ по параметрам $\theta_{n}$ :

$$
\frac{\partial Q_{M}}{\partial \theta_{n}}=2 \sum_{m} r_{m} \frac{\partial r_{m}}{\partial \theta_{n}}=0, n=1, \ldots, N
$$

Для решения системы уравнений (1) применяют итерационную процедуру:

$$
\theta_{n} \approx \theta_{n}^{k+1}=\theta_{n}^{k}+\Delta \theta_{n}, \quad k=0,1, \ldots
$$

В (2) $\theta_{n}^{0}$ - некоторое начальное приближение, $k=0,1, \ldots-$ номер итерации.

На каждой итерации проводят линеаризацию с помощью приближения рядом Тейлора относительно параметров $\theta^{k}$ :

$$
F\left(\mathbf{r}_{m}, \theta\right) \approx y_{m}+\sum_{n} \frac{\partial F\left(\mathbf{r}_{m}, \theta^{k}\right)}{\partial \theta_{n}}\left(\theta_{n}-\theta_{n}^{k}\right) \approx F\left(\mathbf{r}_{m}, \theta^{k}\right)+\sum_{n} J_{m n} \Delta \theta_{n} .
$$


В терминах линеаризованной модели

$$
\frac{\partial F_{m}}{\partial \theta_{n}}=-J_{m n}, q_{m}=\Delta y_{m}-\sum_{n=1}^{N} J_{m n} \Delta \theta_{n}, \text { a } \Delta y_{m}=y_{m}-F\left(\mathbf{r}_{m}, \theta^{k}\right) .
$$

Подставляя (4) в (1), получим:

$$
-2 \sum_{m=1}^{M} J_{m n}\left(\Delta y_{m}-\sum_{s=1}^{N} J_{m s} \Delta \theta_{s}\right)=0, \quad n=1, \ldots N .
$$

Преобразуя (5), получим систему $n$ уравнений, которую называют нормальным уравнением:

$$
\sum_{m=1}^{M} \sum_{s=1}^{M} J_{m n} J_{m s} \Delta \theta_{s}=\sum_{m=1}^{M} J_{m n} \Delta y_{m}, n=1, \ldots, N .
$$

Или в матричном виде:

$$
\left(J^{T} J\right) \Delta \theta=J^{T} \Delta y .
$$

Вектор оптимальных параметров $\theta_{\min }$ находят из нормального уравнения (6), а искомая зависимость определяется как $f=F\left(\mathbf{r}, \theta_{\min }\right)$.

В практических задачах оценка регрессии проводится методом «опорных векторов» [20]. В этом случае параметризация функции регрессии определяется следующим образом:

$$
F(\mathbf{r}, \theta)=\sum_{n=0}^{N} \theta_{n} \varphi_{n}(\mathbf{r})=\theta \varphi(\mathbf{r}),
$$

где $\left\{\varphi_{n}(\mathbf{r})\right\}_{n=1}^{N}$ называется множеством базисных функций. В качестве базисных функций обычно выбирают радиальные функции $\varphi_{n}(\mathbf{r})=e^{-\gamma_{n} \mid \mathbf{r}-c_{n} \|}$, где $\gamma_{n}$ и $c_{n}-$ параметры. Иногда выбирают полиномиальные и сигмоидальные базисные функции [21].

С учетом (7) аппроксимирующая функция определяется выражением

$$
F(\mathbf{r}, \theta)=\sum_{n=1}^{N} \theta_{n} \varphi_{n}(\mathbf{r})=\sum_{n=0}^{N} \theta_{n} e^{-\gamma_{n}\left\|\mathbf{r}-c_{n}\right\|} .
$$

Метод опорных векторов решает задачу условной минимизации функции эмпирического риска [21]:

$$
R_{e m p}=\frac{1}{M} \sum_{m=1}^{M} L_{\varepsilon}\left(f_{m}, F\left(\mathbf{r}_{m}, \theta\right)\right)_{\theta} \min ,
$$

при условии $\|\theta\| \leq C_{0}$, где $C_{0}$ - константа.

Функция $L_{\varepsilon}(f, F)$ называется $\varepsilon$-нечувствительной функцией потерь и определяется следующим соотношением:

$$
L_{\varepsilon}(f, F)=\left\{\begin{array}{c}
|f-F|-\varepsilon, \text { если }|f-F| \geq \varepsilon \\
0 \text { в остальных случаях }
\end{array},\right.
$$

$\varepsilon$ - заданный уровень погрешности.

Подробно метод опорных векторов описан в [20]. 
Отметим, что для нахождения оптимальных параметров нелинейных регрессионных моделей используются также другие численные методы оптимизации, такие как метод сопряженных градиентов, метод Ньютона-Гаусса $[20,21]$ или алгоритм Левенберга-Марквардта $[20,22]$. Нелинейность $F$ в регрессионной модели чаще всего бывает полиномиальной, экспоненциальной или гиперболической.

\section{3 Пример аппроксимации энерговыделения излучения в пористом мате- риале}

С учетом описанной структуры материала исследуемого объекта, расположения пор и детекторов предложен следующий способ локальной аппроксимации по иелевому множеству с применением модели нелинейной регрессии.

- Из целевого множества выбирается произвольная точка $(x, y, z)$, с центром в которой строится сфера радиуса $R$ (вопрос о выборе $R$ рассмотрен ниже).

- Из обучающего множества выбираются точки, находящиеся внутри построенной сферы. С использованием этих точек строится аппроксимирующая функция с применением модели нелинейной регрессии.

- С помощью «обученной» модели нелинейной регрессии рассчитывается отклик (значение энерговыделения) в выбранной точке $(x, y, z)$ целевого множества.

Процесс повторяется для всех точек целевого множества.
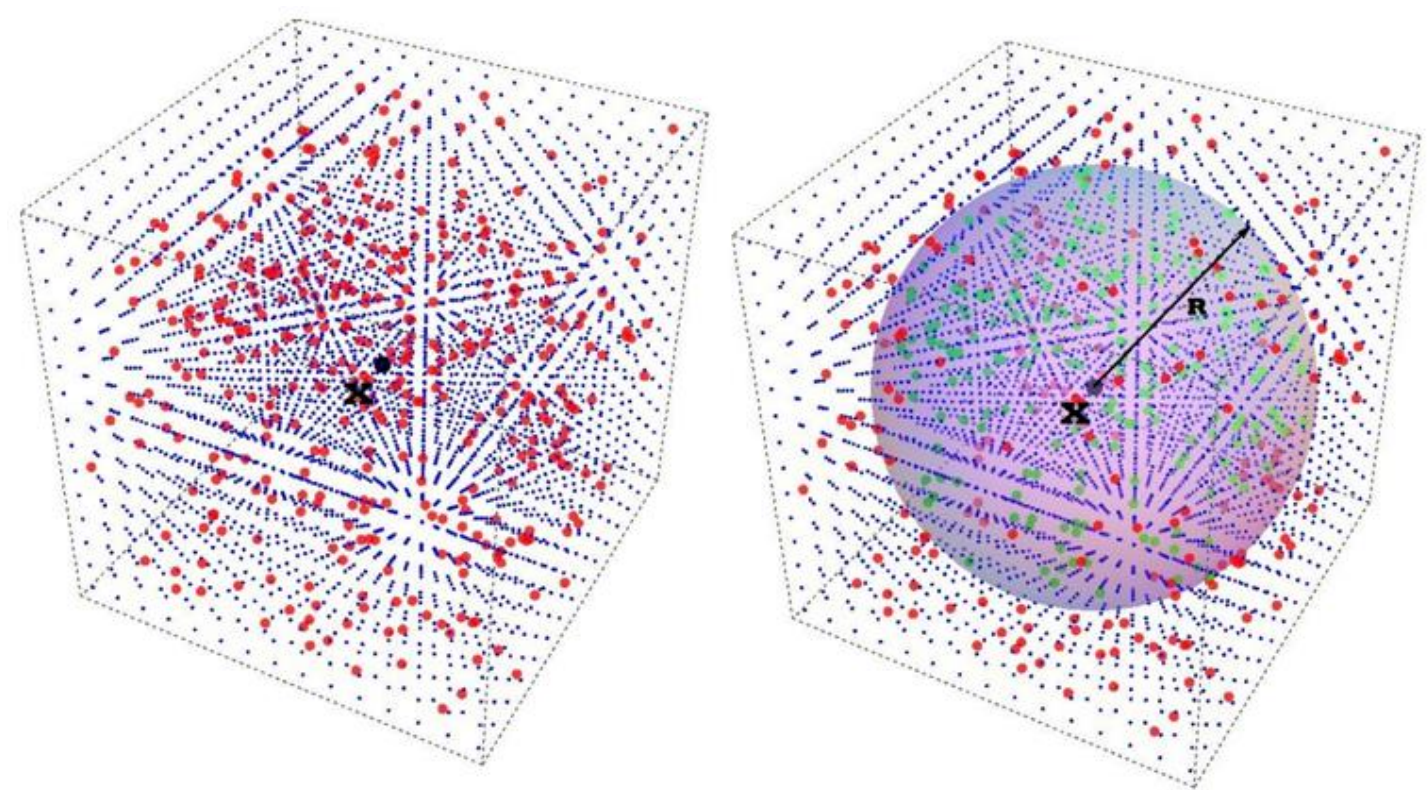

Рис. 6. Построение локальной аппроксимации по целевому множеству

Схема процесса изображена на рис. 6. На этом рисунке синим цветом изображены точки целевого множества в некоторой части объекта; красным точки обучающего множества (слева); зеленым -точки в сфере с центром в выбранной точке целевого множества $\mathbf{X}(x, y, z)$, используемых для построения аппроксимации (справа). 
Для выбора радиуса сферы $R$ применяется следующий подход. Выбирается начальное значение $R$, равное размеру пор, который в данном примере составляет 0.005 см. Далее определяются точки обучающего множества, попавшие в эту сферу. Если в сферу попадает менее 10 точек этого множества, радиус увеличивается на 0.01 до тех пор, пока в сферу не попадет по крайней мере 10 точек обучающего множества. Как показали численные исследования, такое количество точек в сфере вокруг фиксированной точки целевого множества оказывается достаточным для удовлетворительной аппроксимации искомой функции. Указанный способ выбора радиуса $R$ назван «адаптивным».

C применением указанного выше способа аппроксимации были получены приближенные значения энерговыделения в точках термодинамической разностной сетки (целевое множество) $100 \times 100 \times 25$. На рис. 7 изображены полученные приближенные значения энерговыделения в узлах разностной сетки на глубине $z=0.125$ в рассматриваемом объекте.
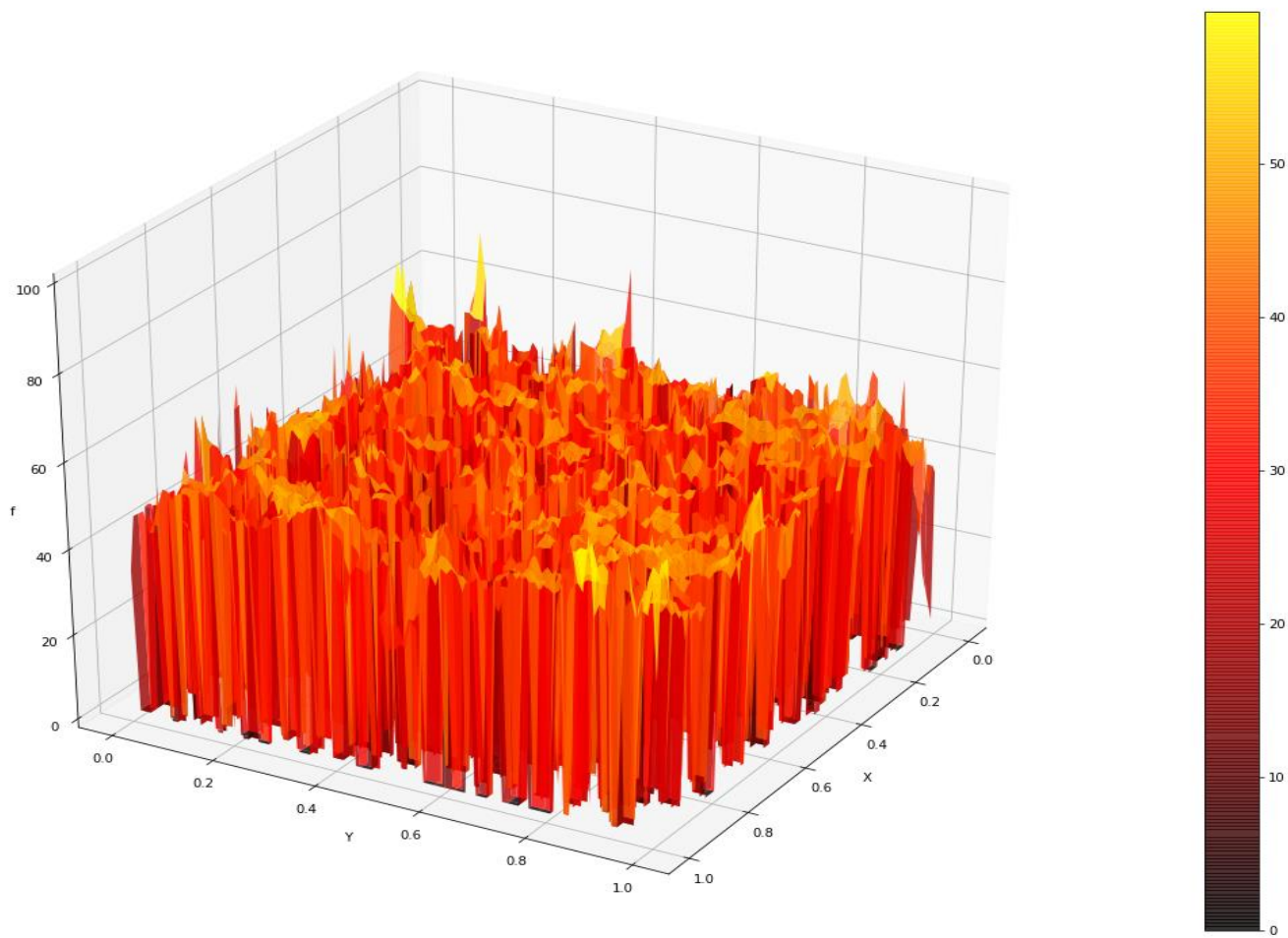

Рис. 7. Результаты аппроксимации при $\mathrm{z}=0.125$

На рис. 7 представлены аналогичные значения, вид сверху.

На рис. 8 можно отчетливо видеть поры (значение энерговыделения в них равно нулю). 


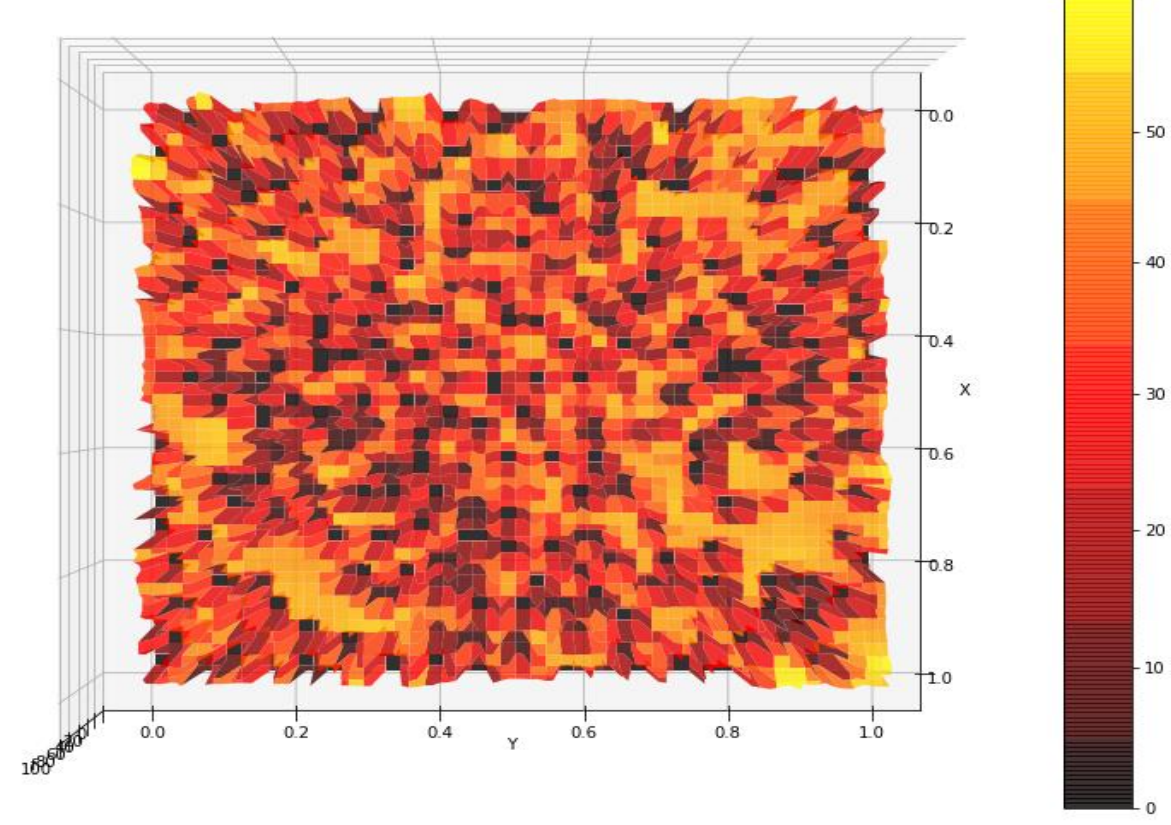

Рис. 8. Результаты аппроксимации. Вид сверху. $Z=0.125$

$3 \mathrm{D}$ изображение энерговыделения в ячейках термодинамической сетки показано на рис. 9.
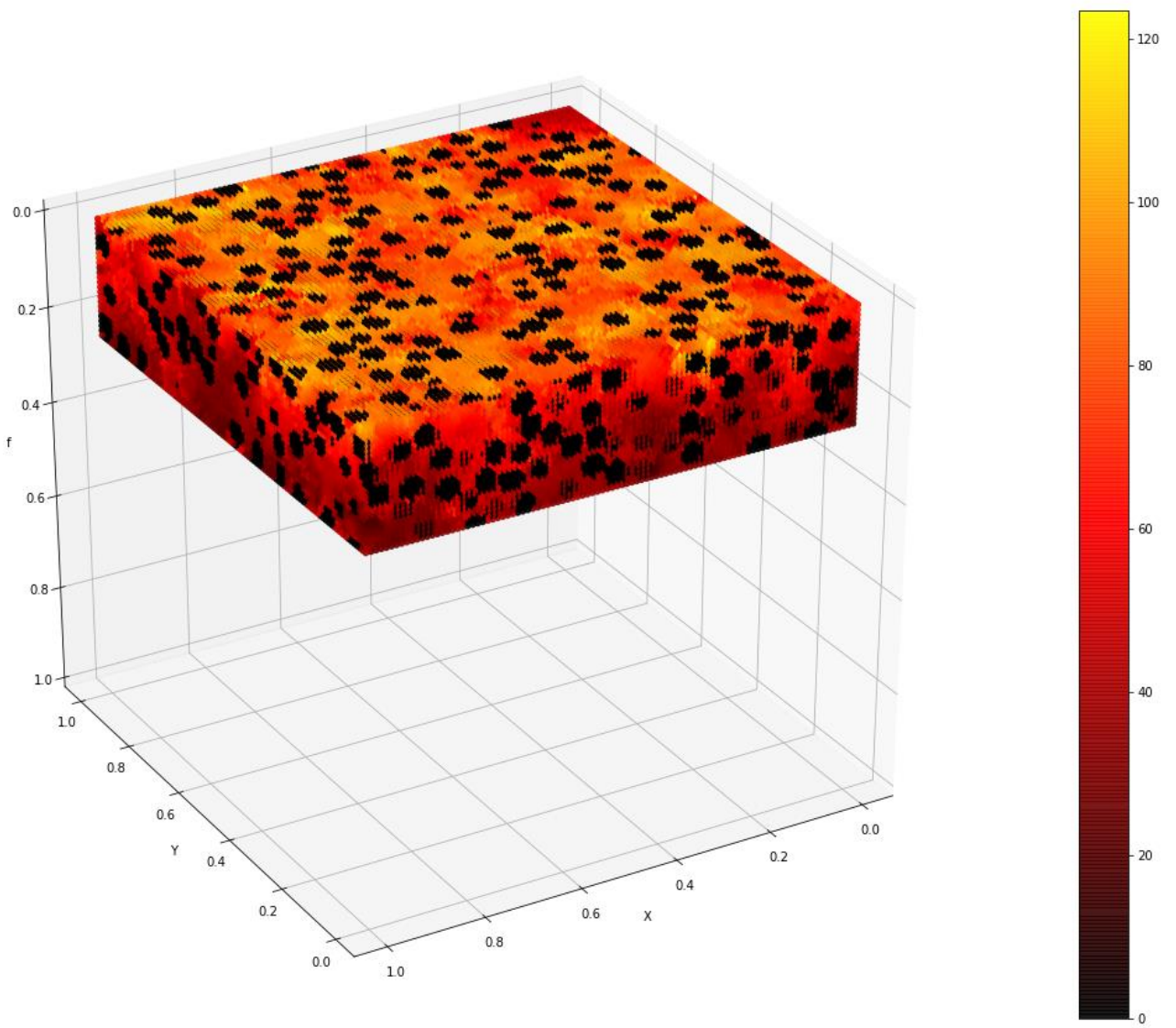

Рис. 9. Результаты аппроксимации энерговыделения в объекте в ячейках термодинамической сетки; черным цветом изображены поры 
На этом рисунке изображены исходный объект и распределение энерговыделения в нем (в относительных единицах).

Отметим, что вычислительный эксперимент проведен в ситуации, когда плоский поток фотонов рентгеновского излучения с энергией 10 кэВ падает на объект перпендикулярно его верхней грани (рис. 9).

\section{3. Результаты моделирования радиационно-индуцированных термомеханических полей}

В настоящем разделе описаны результаты математического трехмерного моделирования радиационно-индуцированных термомеханических эффектов на пористый объект, структура которого описана в разделе 3.

В качестве источника энергии, инициирующего термомеханические процессы, используются значения плотности энерговыделения в ячейках термодинамической разностной сетки, полученные с применением аппроксимации результатов статистического моделирования процесса энерговыделения в объекте, облучаемом рентгеновским излучением (раздел 3 , рис. 8 ).

Основой для расчета термодинамических процессов выбрана идеальная гидродинамическая модель Эйлера динамики сжимаемой однокомпонентной среды в консервативной форме, дополненная уравнением состояния в форме Ми-Грюнайзена.

Для решения системы уравнений идеальной гидродинамики использована консервативная разностная схема повышенного порядка точности [10 - 12]. Интегрирование по времени осуществляется в рамках схемы предиктор-корректор, обеспечивающей второй порядок аппроксимации по времени. На каждом этапе применяется явная схема с ограничением на шаг по времени согласно условию Куранта. Этап корректора в данной схеме служит не только для повышения порядка аппроксимации по времени, но и является дополнительной стабилизирующей процедурой, обеспечивая отсутствие численной антидиффузии, возникающей при обычной явной по времени аппроксимации и приводящей к укручению профилей решений в схемах повышенного порядка точности по пространству.

Ниже приведены результаты модельных расчетов.

На рис. 10, 11 представлены результаты трехмерного моделирования термомеханического воздействия фотонов рентгеновского излучения на пористую структуру из эпоксидной смолы. Эпоксидная смола характеризуется следую-

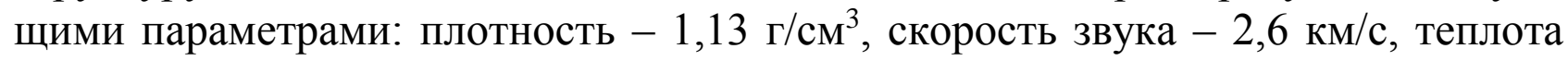
сублимации -2 кДж/г, откольная прочность - 0,33 ГПа, коэффициент Грюнайзена - 0,8. Флюенс энергии фотонов - 25 кал/см².

Расчеты выполнены в декартовой системе координат, в прямоугольном параллелепипеде $0 \mathrm{~mm} \leq \mathrm{x} \leq 1 \mathrm{mM}, 0 \mathrm{mм} \leq \mathrm{y} \leq 1$ мм, $0 \mathrm{~cm} \leq \mathrm{z} \leq 0,25$ мм (рис. 9). Плоскость $\mathrm{z}=0$ соответствует поверхности образца, на которую падает рентгеновское излучение. Направление потока фотонов совпадает с осью z. В области была построена равномерная координатная расчетная сетка с шагом $\mathrm{h}=0.01 \mathrm{mм}$ 
(раздел 3.3), на которую аппроксимированы результаты статистического расчета энерговыделения излучения. Для осуществления сквозного счета в области пор в начальный момент времени добавлен газ с низкой плотностью $-10^{-7}$ $\Gamma / \mathrm{cm}^{3}$.

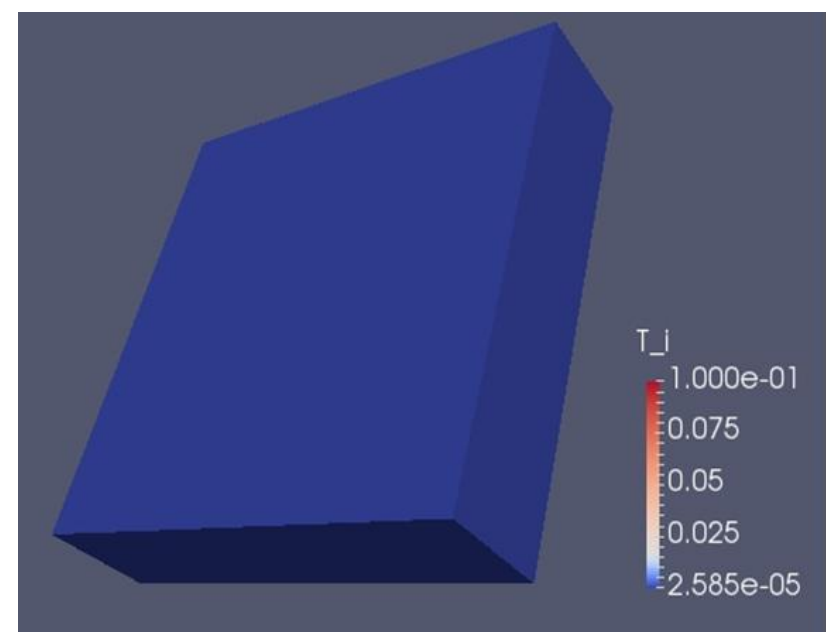

а) до воздействия излучения

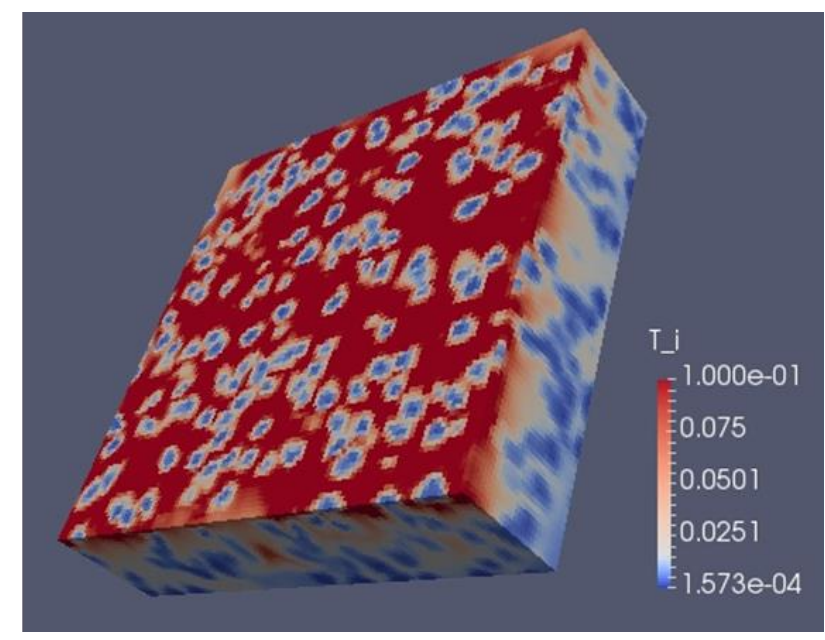

б) после воздействия излучения

Рис. 10. Результаты расчета распределения температуры в объекте, кэВ

В начальный момент времени пористая структура находится в тепловом равновесии при комнатной температуре - рисунок 10a.

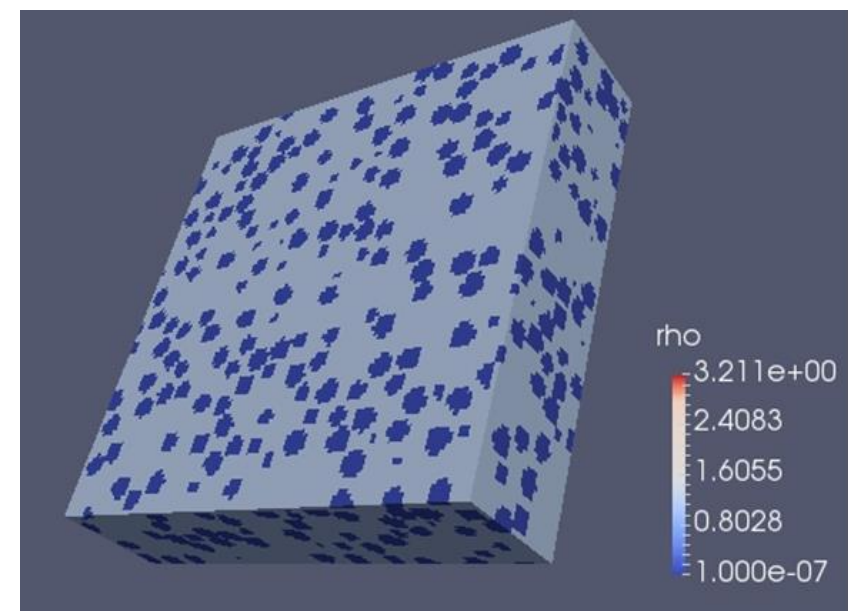

а) до воздействия излучения

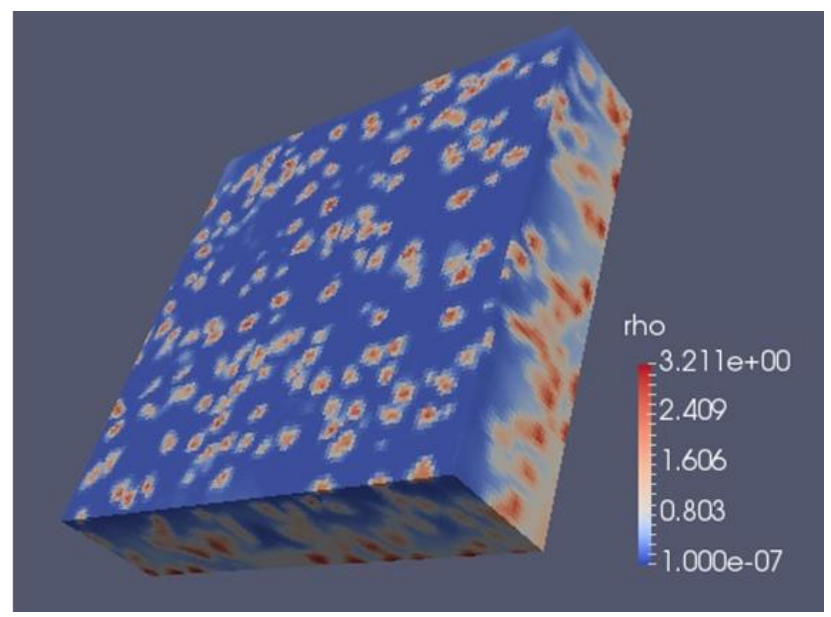

б) после воздействия излучения

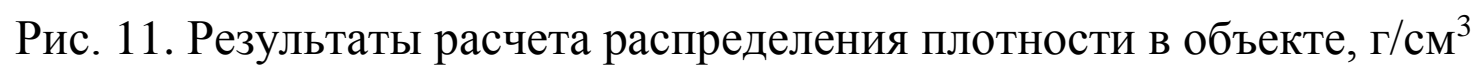

Рис. 11а и $11 б$ показывают, что области пустот в эпоксидной смоле после облучения объекта заполняются веществом. В результате поры превращаются в центры концентрации вещества, что снижает механический импульс ударной волны, распространяющейся вглубь эпоксидной смолы. На рис. 12 представлена зависимость средней плотности вещества в порах от времени. 


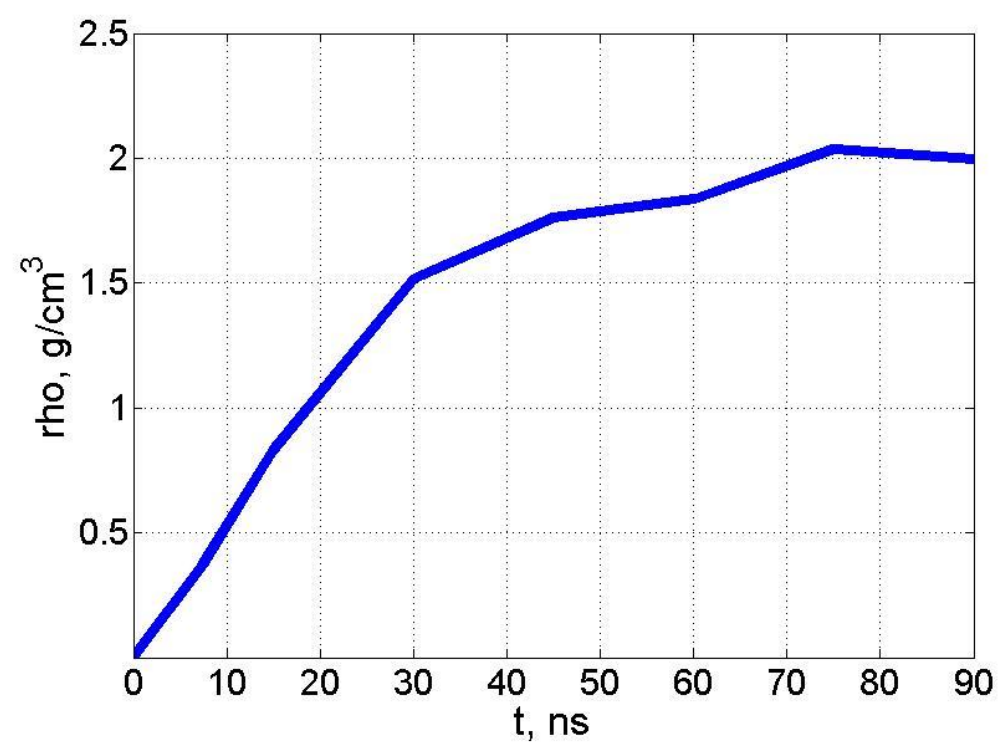

Рис. 12. Зависимость средней плотности вещества в порах от времени

\section{4. Заключение}

Построен способ подготовки исходных данных для моделирования радиационно-индуцированных термомеханических эффектов в гетерогенных материалах пористого типа. Способ основан на многомерной аппроксимации результатов моделирования взаимодействия излучения с веществом на разностную сетку, предназначенную для численного решения уравнений термодинамики. Для построения аппроксимирующей функции применяется технология машинного обучения. В качестве обучающего множества выбирается совокупность точек - центров детекторов и значения плотности энерговыделения в них, а в качестве целевого множества - центры ячеек прямоугольной разностной термодинамической сетки. Показано, что эффективным для вещества пористой структуры является метод локальной по целевому множеству аппроксимации на основе модели нелинейной регрессии.

Проведены предварительные модельные расчеты термомеханических полей в пористом образце. Результаты моделирования показали, что поры после облучения объекта заполняются веществом. В результате поры превращаются в центры концентрации вещества, что снижает механический импульс ударной волны, распространяющейся вглубь образца.

\section{СПИСОК ЛИТЕРАТУРЫ}

1. Pelevin F.V., Avramov N.I., Orlin S.A., Sintsov A.L. Inzhenernyi zhurnal: nauka i innovatsii - Engineering Journal: Science and Innovation, 2013, no. 4. Available at: http://engjournal.ru/catalog/machin/rocket/698.html (March 20, 2016).

2. Хи G., Liи Y., Lио X., Ma J., Li H. Experimental investigation of transpiration cooling for sintered woven wire mesh structures. International Journal of Heat and Mass Transfer, 2015, vol. 91, pp. 898-907. 
3. Bunker R.S. Gas turbine cooling. Moving from macro to micro cooling. Proceedings of the ASME Turbo Expo, 2013, p. 3.

4. Жуковский М.Е., Усков Р.В., Савенков Е.Б., Алексеев М.В., Марков М.Б., Воронин Ф.Н. Модель переноса излучения в веществе гетерогенных материалов пористого типа // Математическое моделирование, 30:10 (2018), 3-20.

5. Zhukovskiy M.E., Podoliako S.V., Markov M.B., Uskov R.V., Bellon C., Jaenisch $G$. $R$. Supercomputing the cascade processes of radiation transport. 19th WCNDT - World conference on nondestructive testing (Proceedings) (2016), http://www.ndt.net/article/wcndt2016/papers/p49.pdf.

6. Жуковский М.Е., Усков Р.В. Гибридное распараллеливание алгоритмов моделирования каскадных процессов переноса излучения // Матем. моделирование, 27:5 (2015), 39-51 http://mi.mathnet.ru/mm3598.

7. Ефремов В.П., Потапенко А.И. Термомеханические процессы в гетерогенных материалах под действием интенсивных потоков энергии // Теплофизика высоких температур. 2010. том 48. № 6. с. 1-7.

8. Zhukovskiy M., Podoliako S., Jaenisch G.-R., Bellon C., Samadurau U. Monte Carlo Simulation Tool with CAD Interface. // Review of Progress in Quantitative Nondestructive Evaluation, Vol. 25, P. 574-579 (2006).

9. Жуковский М.Е., Подоляко С.В., Усков Р.В. Моделирование переноса электронов в веществе на гибридных вычислительных системах // Вычислительные методы и программирование, 2011, т.12, №1, с.152-159.

10. Gasilov V. et al. Towards an Application of High-Performance Computer Systems to 3D Simulations of High Energy Density Plasmas in Z-Pinches // Applications, Tools and Techniques on the Road to Exascale Computing. IOS Press: Advances in Parallel Computing, 2012. Vol. 22, pp. 235-242.

11.Гасилов В.А., Болдарев А.С., Дьяченко С.В., Ольховская О.Г., Карташева Е.Л., Болдырев С.Н., Багдасаров Г.А., Гасилова И.В., Бояров М.С., Шмыров $B . A$. Пакет прикладных программ MARPLE3D для моделирования на высокопроизводительных ЭВМ импульсной магнитоускоренной плазмы // Математическое моделирование, 2012, Т. 24, № 1, с. 55-87.

12. Болдарев А.С. О конечноразностном решении гиперболических систем дифференциальных уравнений с повышенным порядком аппроксимации на двумерных неструктурированных сетках. - М.: ИММ РАН, 1993, препринт №35.

13. Egorova V., Zhukovskiy M. Handling of the radiative electron emission modeling results by use of the neural networks // MATHEMATICA MONTISNIGRI Vol XXXVIII (2017) p. 89-99.

14. Егорова В.А., Иноземцева К.К., Жуковский М.Е. Подход к моделированию радиационно-индуцированных вторичных эффектов в сложных технических 
объектах // Препринты ИПМ им. М.В.Келдыша. 2018. № 110. 24 с. doi:10.20948/prepr-2018-110

http://library.keldysh.ru/preprint.asp?id=2018-110 .

15. Seber G. A. F., Wild C. J. Nonlinear Regression. New York: John Wiley and Sons, 1989.

16. Stillinger F.H., Lubachevsky B.D. Crystalline-Amorphous Interface Packings for Disks and Spheres // J. Stat. Phys. 1993, v,73, №3-4, p.497-514.

17. Lubachevsky B.D., Stillinger F.H. Geometric properties of random disk packings // J. Statistical Physics, 1990, 60, p.561-583.

18. Lubachevsky B.D. How to Simulate Billiards and Similar Systems // Journal of Computational Physics, 1991, v.94, №2, p.255-283.

19. Сквориов А.B. Триангуляция Делоне и её применение. - Томск: Изд-во Томского университета, 2002. - 128 с. - ISBN 5-7511-1501-5.

20. Хайкин $C$. Нейронные сети: полный курс, 2-е издание: Пер. с англ. - М.: Издательский дом «Вильямс», 2006. -1104 с.

21. Васильев Ф. П. Методы оптимизации - Издательство «Факториал Пресс», 2002.

22. Levenberg $K$. Method for the Solution of Certain Problems in Last Squares. Quart. Appl. Math. 1944. Vol. 2. P. 164-168. 